\title{
Purpura fulminans caused by meningococcemia
}

\section{Mukul P. Agarwal MD, Vishal Sharma MD}

A 15-year-old girl presented with a two-day history of fever, confusion and rash. On examination, she had hypotension, stiffness of the neck and a purpuric rash covering her extremities (Figure 1A). A lumbar puncture showed a cloudy cerebrospinal fluid with 4400 cells, 90\% neutrophils, a protein level of 2.4 (normal 0.15-0.5) $\mathrm{g} / \mathrm{L}$ and a glucose level of 0.33 (normal 2.22-3.9) $\mathrm{mmol} / \mathrm{L}$. Gram staining of cerebrospinal fluid showed gram-negative diplococci. Latex agglutination testing was positive for type A meningococcal infection. The patient had not received vaccination for any strain of meningococcus.

The patient was given intravenous fluids, ceftriaxone and hydrocortisone. Blood cultures were positive for Neisseria meningitidis, and the isolates were sensitive to both penicillin and ceftriaxone. After two days of treatment, the patient regained conciousness and her fever subsided. However, the lesions on her skin progressed to symmetric peripheral gangrene (Figure 1B). Autoamputation of five toes eventually occurred. Contacts received a single dose of ciprofloxacin $500 \mathrm{mg}$ as chemoprophylaxis.

Purpura fulminans is a cutaneous manifestation of disseminated intravascular coagulation. It presents as a purpuric rash and symmetric gangrene that often necessitates amputation. It can accompany infections with meningococcus, varicella, Staphylococcus aureus, streptococcus and Hemophilus influenzae. Management includes vigorous fluid resuscitation, vasopressors to alleviate shock and appropriate antibiotics. Hydrocortisone is used for adrenal insufficiency. Fresh frozen plasma and platelet transfusions may be necessary for coagulopathy. Use of activated protein $\mathrm{C}$ can reduce risk of mortality and the need for amputations, although it may increase the risk of intracerebral bleeding in patients with meningitis. ${ }^{1}$ Heparin, along with activated protein $\mathrm{C}$ and hemodiafilteration, may also be of benefit. ${ }^{2}$

Recent clinical guidelines have included recommendations for immunization of this at-risk group and of health care workers as well as chemoprophylaxis of close contacts during meningococcal outbreaks. ${ }^{3.4}$

This article has been peer reviewed.

From the Department of Medicine, University College of Medical Sciences, Delhi, India

CMAJ 2010.DOI:10.1503/cmaj.090103

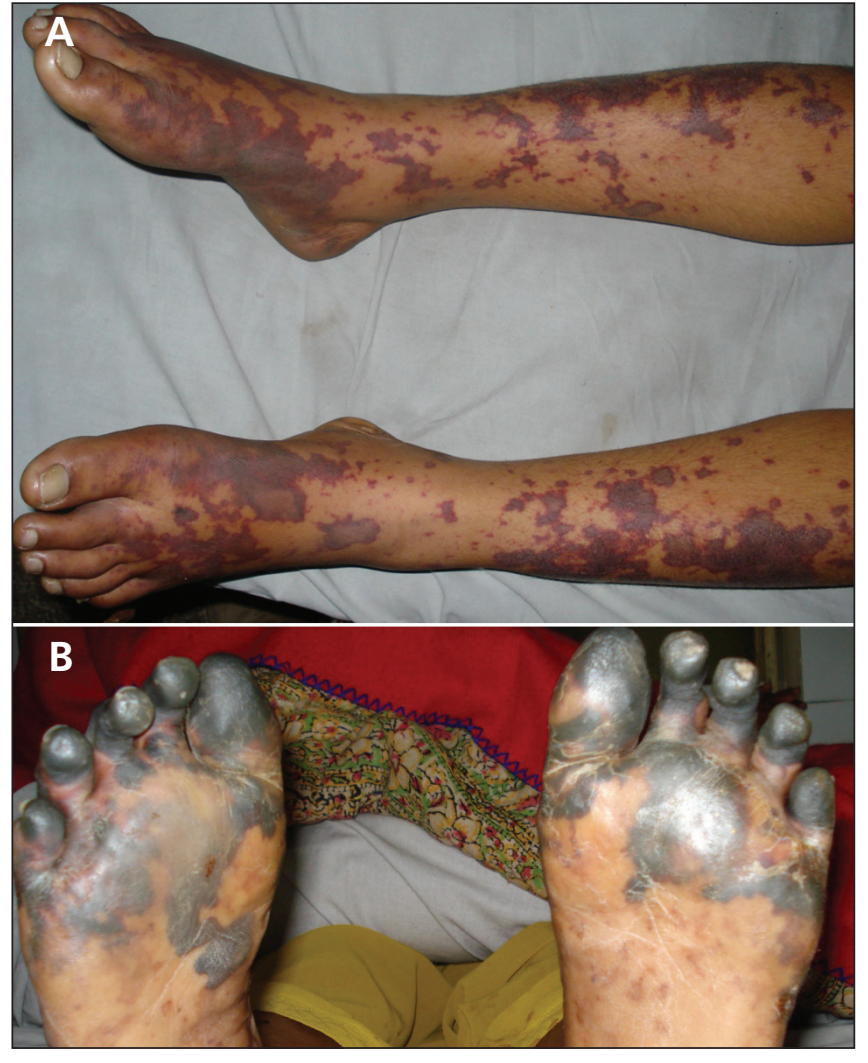

Figure 1: (A) Bilateral purpuric rash on the legs of a 15-year-old girl with disseminated intravascular coagulation associated with meningococcal meningitis. (B) Symmetric gangrene in the patient's feet two weeks later.

Competing interests: None declared.

\section{REFERENCES}

1. White B, Livingstone W, Murphy C, et al. An open-label study of the role of adjuvant hemostatic support with protein $\mathrm{C}$ replacement therapy in purpura fulminansassociated meningococcemia. Blood 2000;96:3719-24.

2. Smith OP, White B, Vaughan D, et al. Use of protein-C concentrate, heparin, and haemodiafiltration in meningococcus-induced purpura fulminans. Lancet 1997;350:1590-3.

3. The Association of Physicians of India. Executive summary. The Association of Physicians of India. Evidence-based clinical practice guidelines on adult immunization [AIP guidelines]. Mumbai (India): The Association; 2009. Available: www.japi.org/april_2009/guideline.pdf (accessed 2009 June 16)

4. National Advisory Committee on Immunization (NACI). Statement on conjugate meningococcal vaccine for serogroups A, C, Y and W135. An Advisory Committee Statement (ACS). Can Commun Dis Rep 2007;33:1-23. 